Research Paper

\title{
Nosocomial candidiasis in Rio de Janeiro State: Distribution and fluconazole susceptibility profile
}

\author{
Paulo Murillo Neufeld ${ }^{1,5}$ Marcia de Souza Carvalho Melhem², Maria Walderez Szeszs², \\ Marcos Dornelas Ribeiro ${ }^{3}$, Efigênia de Lourdes Teixeira Amorim ${ }^{4}$, Manuela da Silva ${ }^{5}$, \\ Marcia dos Santos Lazéra ${ }^{6}$ \\ ${ }^{1}$ Departamento de Análises Clínicas e Toxicológicas, Universidade Federal do Rio de Janeiro, \\ Rio de Janeiro, RJ, Brazil. \\ ${ }^{2}$ Serviço de Parasitologia, Instituto Adolfo Lutz, São Paulo, SP, Brazil. \\ ${ }^{3}$ Serviço de Patologia Clínica, Instituto Estadual de Hematologia Arthur da Siqueira Cavalcante, \\ Rio de Janeiro, RJ, Brazil. \\ ${ }^{4}$ Setor de Microbiologia e Urinálise, Laboratório Sérgio Franco, Rio de Janeiro, RJ, Brazil. \\ ${ }^{5}$ Instituto Nacional de Controle de Qualidade em Saúde, Fundação Oswaldo Cruz, \\ Rio de Janeiro, RJ, Brazil. \\ ${ }^{6}$ Laboratório de Micologia, Instituto de Pesquisa Clínica Hospital Evandro Chagas, \\ Fundação Oswaldo Cruz, Rio de Janeiro, RJ, Brazil.
}

Submitted: August 14, 2012; Approved: December 08, 2014.

\begin{abstract}
One hundred and forty-one Candida species isolated from clinical specimens of hospitalized patients in Rio de Janeiro, Brazil, during 2002 to 2007, were analized in order to evaluate the distribution and susceptibility of these species to fluconazole. Candida albicans was the most frequent species (45.4\%), followed by C. parapsilosis sensu lato (28.4\%), C. tropicalis (14.2\%), C. guilliermondii (6.4\%), C. famata (2.8\%), C. glabrata $(1.4 \%)$, C. krusei $(0.7 \%)$ and C. lambica $(0.7 \%)$. The sources of fungal isolates were blood $(47.5 \%)$, respiratory tract $(17.7 \%)$, urinary tract $(16.3 \%)$, skin and mucous membrane $(7.1 \%)$, catheter $(5.6 \%)$, feces $(2.1 \%)$ and mitral valve tissue $(0.7 \%)$. The susceptibility test was performed using the methodology of disk-diffusion in agar as recommended in the M44-A2 Document of the Clinical and Laboratory Standards Institute (CLSI). The majority of the clinical isolates $(97.2 \%)$ was susceptible (S) to fluconazole, although three isolates $(2.1 \%)$ were susceptible-dose dependent (S-DD) and one of them $(0.7 \%)$ was resistant (R). The S-DD isolates were C. albicans, C. parapsilosis sensu lato and C. tropicalis. One isolate of C. krusei was resistant to fluconazole. This work documents the high susceptibility to fluconazole by Candida species isolated in Rio de Janeiro, Brazil.
\end{abstract}

Key words: Candida, fluconazole, antifungal susceptibility, disk diffusion method.

\section{Introduction}

Since the 1980 decade, invasive fungal infections have grown considerably and the focus of this process has been immunocompromised patients (Beck-Sagué and Jarvis, 1993; Martin et al., 2003). Among the factors that make the defense system fragile and the individuals susceptible to a variety of opportunistic fungi are the seriousness of the base disease, time of permanence in the intensive therapy unit, antibiotic therapy of large spectrum, chemotherapy, radiotherapy, immunosuppressive therapy, central venous catheter, total parenteral feeding, attended ventilation, burns, abdominal surgeries and organ as well as bone marrow transplantations (Colombo and Guimarães, 2003; Colombo et al., 2007; Pemán and Salavert, 2012). Although,

Send correspondence to P.M. Neufeld. Departamento de Análises Clínicas e Toxicológicas, Faculdade de Farmácia, Universidade Federal do Rio de Janeiro, Prédio do CCS, bloco A2, sala 029, Cidade Universitária, 21941-590 Rio de Janeiro, RJ, Brazil. E-mail: pmneufeld@pharma.ufrj.br. 
new fungal species are described each year as agents of nosocomial infection (Chakrabarti and Singh, 2011; Nucci and Marr, 2005), Candida spp. are still considered as the most current pathogen (Alangaden, 2011; Pfaller et al., 2006a). Based on the literature, C. albicans is detected in the majority of the cases in Latin America followed by $C$. tropicalis, C. parapsilosis sensu lato, and C. glabrata (Pfaller et al., 2010). Nevertheless, C. famata, C. kefyr, C. guilliermondii, C. lusitaniae, C. pelliculosa and C. rugosa have presented increasing isolation rates (Colombo et al., 2006; Matta et al., 2007; Pfaller and Diekema, 2007).

Due to the long use for fluconazole to the invasive candidiasis treatment, strains of non-albicans species with low susceptibility have appeared in the hospital ambient (Chen et al., 2012). As the resistance to fluconazole can be the cause of therapeutic failure, the fast identification of the etiologic agent and the analysis of the susceptibility profile to the antifungal drugs can help to decide the most appropriate treatment (Montravers and Jabbour, 2006; Pfaller and Diekema, 2007; Shah et al., 2011).

The method employed to evaluate antifungal susceptibility must present a good clinical correlation and has to be reproducible (Hospenthal et al., 2004; Lass-Flörl et al., 2010). Within this context, an effort has been made by the Clinical and Laboratory Standards Institute - CLSI, which developed a reference test to evaluate the in vitro susceptibility of Candida spp. to fluconazole (CLSI, 2009), using the disk-diffusion in agar methodology (M44-A2). Due to its simplicity, facility of execution and low cost, this method can be easily incorporated in the routine of the public and private clinical laboratories, working as a predictor of clinical response as well as a tool of surveillance and control of the emergence of Candida spp. strains with low sensitivity to fluconazole (Pfaller et al., 2004).

The present study describes the distribution of Candida species isolated from clinical specimens obtained from hospitalized adult and infant patients in Rio de Janeiro, Brazil, and their susceptibility profile to fluconazole employing the agar disk diffusion method described by CLSI (CLSI, 2009).

\section{Material and Methods}

\section{Samples}

One hundred and forty-one nosocomial isolates of Candida spp. (one isolate by patient) were obtained from 2002 to 2007 from five medical centers (a tertiary teaching hospital, a tertiary private hospital, a tertiary militar hospital, a hematological and hemotherapic center and an university pediatric center), one public and one private laboratory of clinical analyses, all of them located in the City of Rio de Janeiro. Despite this localization, the nosocomial samples came from different regions of the State of Rio de Janeiro. In this investigation, the hospital-acquired infections were those that were diagnosed while the patient was hospital- ized in the assistance unit. Candida species were isolated from blood, catheter, gastrointestinal tract, genitourinary tract, skin lesions and mitral valve tissue from adult and infant immunocompromised patients. The identification of Candida isolates and the fluconazole disk diffusion susceptibility testing were performed at the Clinical Mycology Laboratory of Pharmacy College of the Federal University of Rio de Janeiro and at the Mycology Sector of Parasitology Service of Adolfo Lutz Institute, São Paulo, Brazil. All information about the samples were obtained from the data records sent with requests for mycological analyses. However, many of them were incomplete. Thus, it was not possible to classify all samples studied.

\section{Yeast Identification}

All isolates of Candida species were identified based on their morphophysiological characteristics. The cultivation in CHROMagar-Candida medium (Company, France) was performed to confirm the viability and pureness, as well as for a preliminary identification of the isolates by the production of chromogen pigments (green: C. albicans, blue: C. tropicalis and rose: C. krusei) (Odds and Bernaerts, 1994). The observation of the formation of germinative tube in human serum and the production of chlamydospores in cornmeal agar (Oxoid, England) with tween 80 (Reagen, Brazil) was performed in order to identify $C$. albicans (Dalmau, 1929; Taschdjian et al., 1960). The biochemical identification was conducted through the Vitek commercial system (BioMerieux, France) according to the manufacturer recommendation. The isolates were maintained in sterile distilled water at room temperature up to the moment of the susceptibility tests.

\section{Antifungal Susceptibility Testing}

The agar disk-diffusion test was performed in accordance to the methodology described in M44-A2 document published by CLSI (CLSI, 2009). Paper disks containing $25 \mu \mathrm{g}$ of fluconazole (CECON, Brazil) and Petri dishes (90 $\mathrm{mm}$ of diameter) containing Mueller-Hinton agar (Difco, England) supplemented with $2 \%$ of glycose and $0.5 \mu \mathrm{g} \mathrm{mL}^{-1}$ of methylene blue at a depth of $4.0 \mathrm{~mm}$ were used. In order to monitor the precision, accuracy and performance of the test, Candida albicans ATCC 90028 and Candida parapsilosis ATCC 22019 were used as control strains. Each isolate of Candida spp. was subcultivated into plates with Sabouraud dextrose agar (Difco, England), which were incubated at $35-37^{\circ} \mathrm{C}$ for $24 \mathrm{~h}$. Five colonies of the isolates were collected and suspended in $5 \mathrm{~mL}$ of sterile saline $(0.85 \%)$.

The turbidity suspension was adjusted to the $0.5 \mathrm{McF}$ arland scale $\left(10^{6} \mathrm{cell} / \mathrm{mL}\right)$ in a spectrophotometer (Biospectro, Brazil) using $530 \mathrm{~nm}$ wavelength. The yeast suspension was inoculated using a sterile swab over the surface of agar Mueller-Hinton. The disk with fluconazole was aseptically deposited over the inoculated agar and the plate 
was incubated aerobically at a $35-37{ }^{\circ} \mathrm{C}$ temperature for $24 \mathrm{~h}$. The diameter of the inhibition area was measured for determining the susceptibility and calculating the minimal inhibitory concentration (MIC). The interpretative criteria of the fluconazole disk-diffusion test were those suggested by CLSI 7: susceptible (S): $\geq 19 \mathrm{~mm}$; susceptible-dose dependent (S-DD): $15-18 \mathrm{~mm}$; resistant $(\mathrm{R}): \leq 14 \mathrm{~mm}$. The values of the corresponding MIC to these diameters CLSI (CLSI, 2009) are the following: S: MIC $\leq 8 \mu \mathrm{g} \mathrm{mL}^{-1}$; S-DD: MIC $16-32 \mu \mathrm{g} \mathrm{mL}^{-1}$; R: MIC $\geq 64 \mu \mathrm{g} \mathrm{mL}^{-1}$. The quality control test was conducted every day during the procedure according to CLSI (CLSI, 2009) and the results obtained were within expected limit for each control strain.

\section{Results}

The distribution of Candida species at the seven health institutions involved in this investigation is presented in Table 1. From the total of isolations, $43.3 \%$ occurred in the Hemotherapy and Hematology Center. The largest percentage of recovery of Candida spp. from blood stream infections $(46.3 \%)$ was also observed in this same institution. In the present study, Candida albicans (Table 1) was the yeast with the largest isolation rate (45.4\%), followed by C. parapsilosis sensu lato (28.4\%), C. tropicalis (14.2\%), C. guilliermondii (6.4\%) and C. famata (2.8\%), besides C. glabrata (1.4\%), C. krusei $(0.7 \%)$ and $C$. lambica $(0.7 \%)$. The isolates of Candida here evaluated were obtained from hospitalized patients with different risk factors, among them, onco-hematological diseases, solid tumors and AIDS.

Blood $(47.5 \%)$, respiratory tract $(17.7 \%)$, urinary tract $(16.3 \%)$, skin and mucous membrane $(7.1 \%)$, catheter $(5.6 \%)$, feces $(2.1 \%)$ and biopsy of the mitral valve $(0.7 \%)$ were the sources of isolation. It was not possible to define anatomical localization for 3.0\% of the isolates. The distribution of Candida isolates by clinical specimens is summarized in the Table 2. C. albicans and C. parapsilosis sensu lato were the most frequent species isolates from hemoculture, presenting rates of $41.8 \%$ and $37.3 \%$, respectively. Other yeasts isolated from hemoculture were $C$. tropicalis (10.4\%), C. guilliermondii (6.0\%), C. famata (3.0\%) and C. glabrata $(1.5 \%)$. From samples of catheter only C. albicans $(62.5 \%)$ and C. parapsilosis sensu lato (37.5\%) were isolated. The single isolate of $C$. krusei was retrieved from respiratory specimens.

The diameters of inhibition zones produced by the agar disk-diffusion for all of the isolates tested varied from 10 to $50 \mathrm{~mm}$ with average value of $35.8 \mathrm{~mm}$. Table 3 presents the intervals and the means of the diameters from the inhibition zone for Candida species regarding the relationship to different anatomical sites. Among the species, $C$. krusei $(10 \mathrm{~mm})$ presented the smallest inhibition diameter. C. albicans $(38.6 \mathrm{~mm})$, C. parapsilosis sensu lato (36.5 $\mathrm{mm}$ ) and C. guilliermondii $(33 \mathrm{~mm})$ were the species

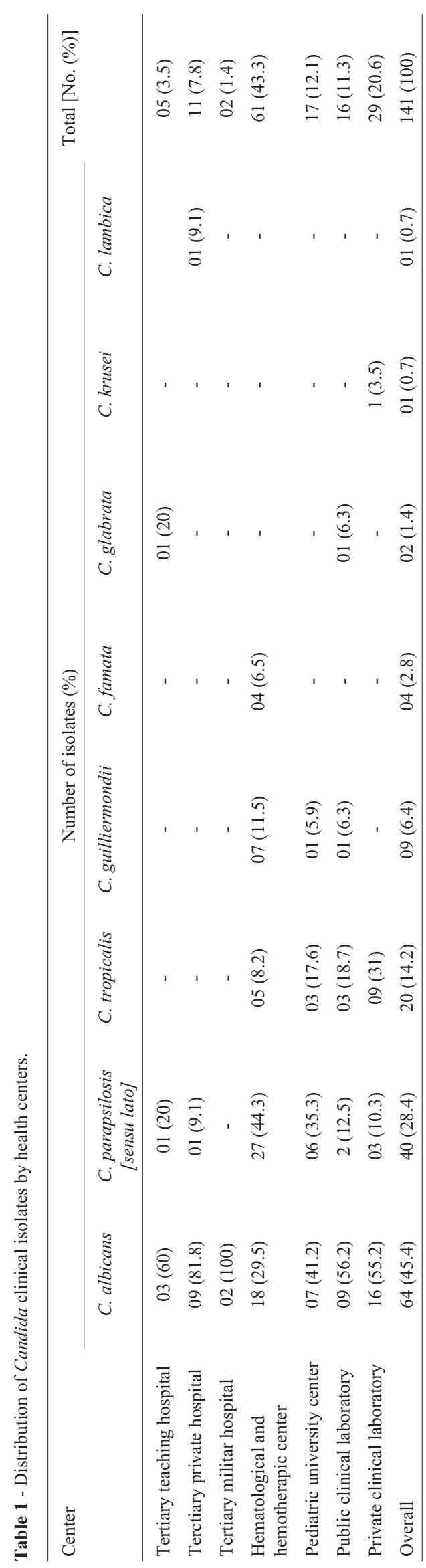



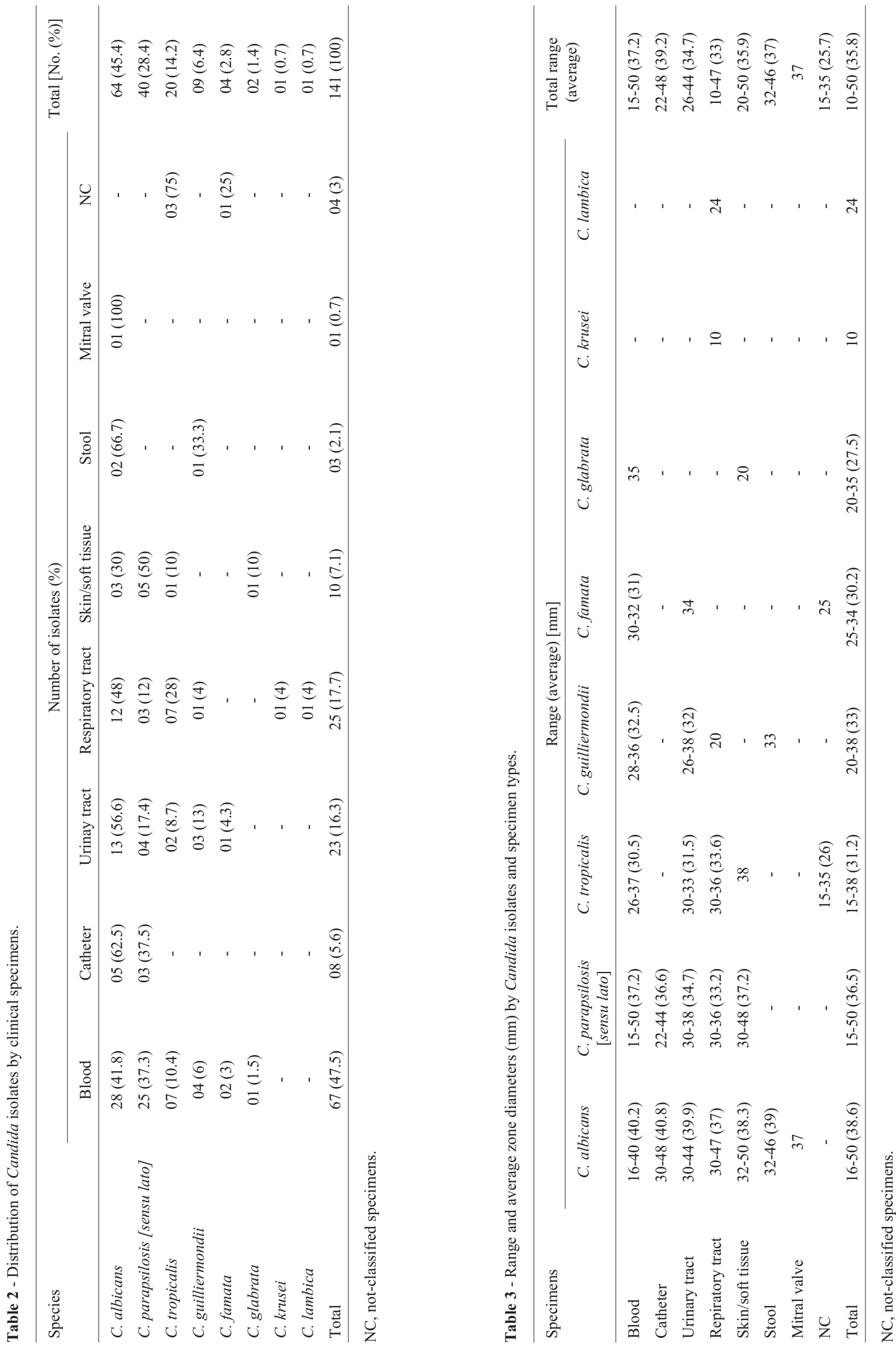
Table 4 - Fluconazole susceptibility of 141 Candida clinical isolates.

\begin{tabular}{|c|c|c|c|c|}
\hline \multirow[t]{2}{*}{ Species } & \multirow{2}{*}{$\begin{array}{c}\text { Clinical } \\
\text { specimens }\end{array}$} & \multicolumn{3}{|c|}{ Fluconazole susceptibility category (\%) } \\
\hline & & S & S-DD & $\mathrm{R}$ \\
\hline \multirow[t]{3}{*}{ C. albicans } & Blood & 96.4 & 3.6 & - \\
\hline & Non-blood & 100 & - & - \\
\hline & All & 98.4 & 1.6 & - \\
\hline \multirow{3}{*}{$\begin{array}{l}\text { C. parapsilosis } \\
\text { [sensu lato] }\end{array}$} & Blood & 96 & 4.0 & - \\
\hline & Non-blood & 100 & - & - \\
\hline & All & 97.5 & 2.5 & - \\
\hline \multirow[t]{4}{*}{ C. tropicalis } & Blood & 100 & - & - \\
\hline & Non-blood & 100 & - & - \\
\hline & $\mathrm{NC}$ & 66.7 & 33.3 & - \\
\hline & All & 95 & 5 & - \\
\hline \multirow[t]{3}{*}{ C. guilliermondii } & Blood & 100 & - & - \\
\hline & Non-blood & 100 & - & - \\
\hline & All & 100 & - & - \\
\hline \multirow[t]{4}{*}{ C. famata } & Blood & 100 & - & - \\
\hline & Non-blood & 100 & - & - \\
\hline & $\mathrm{NC}$ & - & - & - \\
\hline & All & 100 & - & - \\
\hline \multirow[t]{3}{*}{ C. glabrata } & Blood & 100 & - & - \\
\hline & Non-blood & 100 & - & - \\
\hline & All & 100 & - & - \\
\hline \multirow[t]{3}{*}{ C. krusei } & Blood & - & - & - \\
\hline & Non-blood & - & - & 100 \\
\hline & All & - & - & 100 \\
\hline \multirow[t]{3}{*}{ C. lambica } & Blood & - & - & - \\
\hline & Non-blood & 100 & - & - \\
\hline & All & 100 & - & - \\
\hline Overall & & 97.2 & 2.1 & 0.7 \\
\hline
\end{tabular}

S, susceptible; S-DD, susceptible-dose dependent; R, resistant; NC, notclassified specimens.

that produced diameters with higher average values. Based on diameters size of the inhibition zone, the general profile of susceptibility to fluconazole was the following: $0.7 \%$ of the isolates were resistant ( $\mathrm{MIC} \geq 64 \mu \mathrm{g} \mathrm{mL}^{-1}$ ), $2.1 \%$ were susceptible-dose dependent (MIC 16-32 $\mu \mathrm{g} \mathrm{mL}^{-1}$ ) and 97.2\% were susceptible (MIC $\leq 8 \mu \mathrm{g} \mathrm{mL}^{-1}$ ). Table 4 shows the susceptibility to fluconazole separately by species. The resistance to fluconazole was a phenomenon restricted to the only isolate of $C$. krusei in the present investigation. $C$. albicans, C. parapsilosis sensu lato and C. tropicalis, although clearly susceptible, also were susceptible-dose dependent isolates. The other species, C. famata, C. glabrata, C. guilliermondii and C. lambica presented susceptibility rates of $100 \%$.

\section{Discussion}

From the medical centers analyzed, the one specialized in the treatment of hematological patients was the center that presented the largest rate of fungal isolation, including isolates from hemocultures $(46.3 \%)$. Because of its consumptive nature and its more aggressive treatment protocols, the hematological pathologies, in general, make the patients extremely susceptible to invasive fungal infections, therefore becoming one of the major risk factors (Wisplinghoff et al., 2003b). High percentages of fungal recovery from these institutions (or hematological unit) are to be expected (Martin et al., 2003). Within the group of tertiary hospitals and the Pediatric Care Center, the percentages of positive hemoculture were $6.0 \%$ and $13.4 \%$, respectively. Similar rates have been documented by different authors (Velasco and Bigni, 2008; Velasco et al., 2000; Wisplinghoff et al., 2003a). Based on these isolations, nowadays the genus Candida is considered the third more frequent pathogen in infections of stream blood, after Staphylococcus epidermidis and S. aureus (Wisplinghoff et al., 2003b, 2004).

The results here documented highlight the high incidence of C. albicans in hospitalized patients. In the present work, C. albicans represented $41.8 \%$ of all isolates from the hemoculture. This percentage agrees with the data recently pointed out regarding this species $(48.7 \%)$ by the International Program of Epidemiologic Surveillance (SENTRY), which investigated the distribution of Candida species isolated from candidemias and their susceptibility profile to antifungics in North America, Latin America and Europe (Messer et al., 2006). In Brazil, C. albicans has been equally the most isolated yeast in candidemias in many regions of the country (Aquino et al., 2005; Barberino et al., 2006; Passos et al., 2007). The explanation for the fact that C. albicans presents the highest percentage of recovery may be related to its large adaptability and pathogenic versatility (Kumamoto and Vinces, 2005).

In spite of $C$. albicans being the most frequent agent in this investigation (41.8\%), as a whole, the non- $C$. albicans Candida species represented the majority of the isolates $(58,2 \%)$ with preponderancy of $C$. parapsilosis sensu lato (37.3\%) and C. tropicalis (10.4\%). Currently, $C$. parapsilosis sensu lato and C. tropicalis correspond conjunctly to about $70 \%$ of the non-C. albicans Candida isolates from Brazilian candidemias (Colombo et al., 2006). The progressive increase in the rates of recovery of non- $C$. albicans Candida species has been widely related (Bassetti et al., 2011; Pfaller et al., 2010). In Brazil, this tendency was equally confirmed (Nucci et al., 2010; SampaioCamargo et al., 2010) and isolation rates up to $75 \%$ have been reported to non- $C$. albicans Candida species (Pasqualotto et al., 2005). The emergence of non- $C$. albicans Candida isolates may be due to the selection of more resistant strains because of ostensive use of azoles derivatives (Mario et al., 2012). 
In this investigation, blood was the main source of Candida species (47.5\%). Nevertheless, the respiratory tract $(17.7 \%)$, urinary tract $(16.3 \%)$, skin and mucous (7.1\%) also contributed as important sources. In accordance to the findings of Comert et al. (2006), C. parapsilosis sensu lato, $C$. tropicalis and $C$ guilliermondii in the present study were also the main non-C. albicans Candida species recovered from non-sterile specimens. In spite of the hemoculture being the main marker of invasive infection (Martin et al., 2003) and the non-sterile specimens being considered of relative importance to the infection diagnostic (Wang et al., 2004), the isolation of Candida spp. from these specimens may present certain predictive value for candidemias (Sandford et al., 1980), because many invasive processes are associated to the host's microbiota (Agvald-Öhman et al., 2007).

The invasive infections represent high cost to human economy. In the United States the annual cost with these infections is about US\$ 17 billions (Martin et al., 2003). For the candidemia treatment, a cost of about US $\$ 1$ billion per year is estimated (Pfaller et al., 2006b). Among the different factors that contribute in a critical way to calculate economical and social costs of candidemias, it is the microbial resistance to the antifungics of clinical use (Pfaller et al., 2006b, 2007). As a result, the demand for susceptibility tests has been growing (Pfaller, 2012). The agar disk diffusion method may be useful for the selection of more effective, non-toxic and less expensive therapies (Pfaller et al., 2007; Shah et al., 2011). Its effectiveness in the confirmation of the susceptibility or in the detection of resistance to fluconazole has been evaluated in various multicentric international studies (Pfaller et al., 2005, 2007). Good levels of agreement ( 87.4 to $97 \%$ ) between zone diameters by disk-diffusion method and MIC by CLSI reference microdilution method has been found (Barry et al., 2002; Rodero et al., 2006), suggesting an in vivo/in vitro correlation equivalent to the reference method (Meis et al., 2000).

In the present study it was demonstrated the low percentage $(0.7 \%)$ of resistance to fluconazole, corroborating with what has been reported previously by other authors (Azevedo, 2011; Pfaller et al., 2003, 2004). The only resistant isolate was $C$. krusei that was recovered from the respiratory tract. The resistance of this yeast was expected, since it is considered intrinsically resistant to fluconazole (Comert et al., 2006). In spite of the occurrence of susceptibility-dose dependent (2.1\% from the total of isolates), $98.4 \%$ of C. albicans isolates, $97.5 \%$ of C. parapsilosis sensu lato isolates and $95.0 \%$ of C. tropicalis isolates were susceptible to fluconazole. Pfaller et al. $(2005,2007)$ also reported similar rates from global studies, which included the Latin America and Brazil. To C. famata, C. glabrata, C. guilliermondii and C. lambica, the fluconazole was $100 \%$ effective. Nevertheless, acquired resistance within isolates of C. glabrata has been reported (Colombo et al., 2006; Matta et al., 2007). The high susceptibility of Candida iso- lates to fluconazole described here is also very relevant, since this antifungal medicine is the main available azole drug to treat invasive fungal infections within Brazilian public hospitals (Brasil, 2007). The simplicity and validity of the use of the disk-diffusion method were also confirmed.

The study on resistance to fluconazole and other antifungals comparing CLSI disk-diffusion method to CLSI microdilution method and to EUCAST method was conducted and the results will be published soon, as well as the results of the study comparing the genotypic and phenotypic identification of these isolates.

\section{References}

Agvald-Öhman C, Klingspor L, Hjelmqvist H et al. (2007) Invasive candidiasis in long-term patients at a multidisciplinary intensive care unit: Candida colonization index, risk factors, treatment and outcome. Scand J Infect Dis 31:1-9.

Alangaden GJ (2011) Nosocomial fungal infections: epidemiology, infection control, and prevention. Infect Dis Clin North Am 25:201-225.

Aquino VR, Lunardi LW, Goldani LZ et al. (2005) Prevalence, susceptibility profile for fluconazole and risk factors for candidemia in a tertiary care hospital in southern Brazil. Braz J Infect Dis 9:411-4118.

Azevedo AC, Bizerra FC, da Matta DA et al. (2011) In vitro susceptibility of a large collection of Candida Strains against fluconazole and voriconazole by using the CLSI disk diffusion assay. Mycopathologia 171:411-416.

Barberino MG, Silva N, Rebouças C et al. (2006) Evaluation of blood stream infections by Candida in three tertiary hospitals in Salvador, Brazil: a case-control study. Braz J Infect Dis 10:36-40.

Barry AL, Pfaller MA, Rennie RP et al. (2002) Precision and accuracy of fluconazole susceptibility tests by broth microdilution, Etest and disk diffusion methods. Antimicrob Agents Chemother 46:1781-1784.

Bassetti M, Taramasso L, Nicco E et al. (2011) Epidemiology, species distribution, antifungal susceptibility and outcome of nosocomial candidemia in a tertiary care hospital in Italy. PLoS One 6:e24198.

Beck-Sagué CM, Jarvis WR, National Nosocomial Infections Surveillance System (1993) Secular trends in the epidemiology of nosocomial fungal infections in the United States, 1980-1990. J Infect Dis 167:1247-1251

Brasil, Ministério da Saúde. Secretaria de Ciência, Tecnologia e Insumos Estratégicos. Departamento de Assistência Farmacêutica e Insumos Estratégicos (2007) Relação Nacional de Medicamentos Essenciais: RENAME / Ministério da Saúde, Secretaria de Ciência, Tecnologia e Insumos Estratégicos, Departamento de Assistência Farmacêutica e Insumos Estratégicos. $5^{\mathrm{a}}$. ed. Brasília: Editora do Ministério da Saúde. 286 p.: il. (Série B. Textos Básicos de Saúde).

Chakrabarti A, Singh R (2011) The emerging epidemiology of mould infections in developing countries. Curr Opin Infect Dis 24:521-526.

Chen TC, Chen YH, Chen YC et al. (2012) Fluconazole exposure rather than clonal spreading is correlated with the emer- 
gence of Candida glabrata with cross-resistance to triazole antifungal agents. Kaohsiung J Med Sci 28:306-315.

CLSI - Clinical and Laboratory Standards Institute (2009) Publication M44-A2: Method for Antifungal Disk Diffusion Susceptibility Testing of Yeasts; Approved Guideline - Second Edition. Wayne, PA: CLSI 29:1-25.

Colombo AL, Nucci M, Park BJ et al. (2006) Epidemiology of candidemia in Brazil: a nationwide sentinel surveillance of candidemia in eleven medical centers. J Clin Microbiol 44:2816-2823.

Colombo AL, Guimarães T (2003) Epidemiologia das infecções hematogênicas por Candida spp. Rev Soc Br Med Trop 36:599-607.

Colombo AL, Guimarães T, Silva LR et al. (2007) Prospective observational study of candidemia in São Paulo, Brazil: incidence rate, epidemiology, and predictors of mortality. Infect Control Hosp Epidemiol 28:570-576.

Comert F, Kulah C, Aktas E et al. (2006) Identification of Candida species isolated from patient in intensive care unit and in vitro susceptibility to fluconazole for a 3 -year period. Mycoses 50:52-57.

Dalmau LM (1929) Observations on mycological technique with particular references to pathogenic fungi. Porto Rico J Pub Heath Trop Med 05:302-311.

Hospenthal DR, Murray CK, Rinaldi MG (2004) The role of antifungal susceptibility testing in the therapy of candidiasis. Diagn Microbiol Infect Dis 48:153-160.

Kumamoto CA, Vinces MD (2005) Contributions of hyphae and hypha-co-regulated genes to Candida albicans virulence. Cell Microbiol 7:1546-1554.

Lass-Flörl C, Perkhofer S, Mayr A (2010) In vitro susceptibility testing in fungi: a global perspective on a variety of methods. Mycoses 53:1-11.

Mario DA, Denardi LB, Bandeira LA et al. (2012) The activity of echinocandins, amphotericin B and voriconazole against fluconazole-susceptible and fluconazole-resistant Brazilian Candida glabrata isolates. Mem Inst Oswaldo Cruz 107:433-436.

Martin GS, Mannino DM, Eaton S et al. (2003) The Epidemiology of sepsis in the United States from 1979 through 2000. N Engl J Med 348:1546-1554.

Matta DA, Almeida LP, Machado AM et al. (2007) Antifungal susceptibility of 1000 Candida bloodstream isolates to 5 antifungal drugs: Results of a multicenter study conducted in São Paulo, Brazil, 1995-2003. Diagn Microbiol Infect Dis 57:399-404.

Meis J, Petrou M, Bille J et al. (2000) A global evaluation of the susceptibility of Candida species to fluconazole by disk diffusion. Global Antifungal Surveillance Group. Diagn Microbiol Infect Dis 36:215-223.

Messer SA, Jones RN, Fritsche TR (2006) International surveillance of Candida spp. and Aspergillus spp.: report from the SENTRY Antimicrobial Surveillance Program (2003). J Clin Microbiol 44:1782-1787.

Montravers P, Jabbour K (2006) Clinical consequences of resistant Candida infections in intensive care. Int $\mathrm{J}$ Antimicrob Agents 27:1-6.

Nucci M, Queiroz-Telles F, Tobón AM et al. (2010) Epidemiology of opportunistic fungal infections in Latin America. Clin Infect Dis 51:561-570.
Nucci M, Marr KA (2005) Emerging fungal diseases. Clin Infect Dis 41:521-526.

Odds FC, Bernaerts R (1994) CHROMagar Candida, a new differential isolation medium for presumptive identification of clinically important Candida species. J Clin Microbiol 32:1923-1929.

Pasqualotto AC, Nedel WL, Machado TS et al. (2005) Risk factors and outcome for nosocomial breakthrough candidaemia. J Infec 60:129-134.

Passos XS, Costa CR, Araújo CR et al. (2007) Species distribution and antifungal susceptibility patterns of Candida spp. bloodstream isolates from a Brazilian tertiary care hospital. Mycopathologia 163:145-151.

Pemán J, Salavert M (2012) Epidemiología general de la enfermedad fúngica invasora. Enferm Infecc Microbiol Clin 30:90-98.

Pfaller MA (2012) Antifungal drug resistance: mechanisms, epidemiology, and consequences for treatment. Am J Med 125:S3-S13.

Pfaller MA, Boyken L, Hollis RJ et al. (2006a) In vitro susceptibilities of Candida spp. to caspofungin: four years of global surveillance. J Clin Microbiol 44:760-763.

Pfaller MA, Boyken L, Messer SA et al. (2005) Comparison of results of voriconazole disk diffusion testing for Candida species with results from a central reference laboratory in the ARTEMIS global antifungal surveillance program. J Clin Microbiol 43:5208-52013.

Pfaller MA, Diekema DJ (2007) Epidemiology of invasive candidiasis: a persistent public health problem Clin Microbiol Rev 20:133-163.

Pfaller MA, Diekema DJ, Gibbs DL et al. (2010) Results from the ARTEMIS disk global antifungal Surveillance study, 1997 to 2007: a 10.5 year analysis of susceptibilities of Candida species to fluconazole and voriconazole as determined by CLSI standardized disk diffusion. J Clin Microbiol 48:1366-1377.

Pfaller MA, Diekema DJ, Gibbs DL et al. (2007) Results from the ARTEMIS disk global antifungal surveillance study, 1997 to 2005: an 8.5-year analysis of susceptibilities of Candida species and other yeast species to fluconazole and voriconazole determined by CLSI standardized disk diffusion testing. J Clin Microbiol 45:1735-1745.

Pfaller MA, Diekema DJ, Messer SA et al. (2003) Activities of fluconazole and voriconazole against 1.586 recent clinical isolates of Candida species determined by broth microdilution disk diffusion and e-test methods: report from the ARTEMIS global antifungal susceptibility program 2001. J Clin Microbiol 41:1440-1446.

Pfaller MA, Diekema DJ, Sheehan DJ (2006b) Interpretive breakpoints for fluconazole and Candida revisited: a blueprint for the future of antifungal susceptibility testing. Clin Microbiol Rev 19:435-447.

Pfaller MA, Hazen KC, Messer SA et al. (2004) Comparison of results of fluconazole disk diffusion testing for Candida species with results from a central reference laboratory in the ARTEMIS global antifungal surveillance program. J Clin Microbiol 42:3607-3612.

Rodero L, Córdoba S, Vivot W et al. (2006) Método de difusión con discos para la determinación de sensibilidad a fluconazol en aislamientos de Candida spp. Rev Argent Microbiol 38:155-163. 
Sampaio-Camargo TZ, Marra AR, Silva CV et al. (2010) Secular trends of candidemia in a tertiary care hospital. Am J Infect Control 38:546-551.

Sandford GR, Merz WG, Wingard JR et al. (1980) The value of fungal surveillance cultures as predictors of sistemic fungal infections. J Infect Dis 142:503-509.

Shah DN, Yau R, Weston J et al. (2011) Evaluation of antifungal therapy in patients with candidaemia based on susceptibility testing results: implications for antimicrobial stewardship programmes. J Antimicrob Chemother 66:2146-2151.

Taschdjian CL, Burchall JJ, kozinn PJ (1960) Rappid identification of Candida albicans by filamentation on serun and serun substitutes. Am J Dis Children 99:212-215.

Velasco E, Bigni R (2008) A prospective cohort study evaluating the prognostic impact of clinical characteristics and comorbid conditions of hospitalized adult and pediatric cancer patients with candidemia. Eur J Clin Microbiol Infect Dis 27:1071-1078.

Velasco E, Thuler LC, Martins CA et al. (2000) Epidemiology of bloodstream infections at a cancer center. São Paulo Med J 118:131-138.
Wang JL, Chang SC, Hsueh PR et al. (2004) Species distribution and fluconazole susceptibility of Candida clinical isolates in a medical center in 2002. J Microbiol Immunol Infect 37:236-241.

Wisplinghoff H, Bischoff T, Tallent SM et al. (2004) Nosocomial bloodstream infections in US hospitals: analysis of 24,179 cases from a prospective nationwide surveillance study. Clin Infect Dis 39:309-317.

Wisplinghoff H, Seifert H, Tallent SM et al. (2003a) Nosocomial bloodstream infections in pediatric patients in United States hospitals: epidemiology, clinical features and susceptibilities. Pediatr Infect Dis J 22:686-691.

Wisplinghoff H, Seifert H, Wenzel RP et al. (2003b) Current trends in the epidemiology of nosocomial bloodstream infections in patients with hematological malignancies and solid neoplasms in hospitals in the United States. Clin Infect Dis 36:1103-1110.

Associate Editor: Carlos Pelleschi Taborda

All the content of the journal, except where otherwise noted, is licensed under a Creative Commons License CC BY-NC. 